\title{
ESTABLISHMENT AND VALIDATION OF A NOVEL FUCCI (FLUORESCENT UBIQUITINATION-BASED CELL CYCLE INDICATOR) SYSTEM IN AN IN VITRO CHRONIC MYELOID LEUKEMIA MODEL
}

\author{
A Thesis \\ presented to \\ the Faculty of California Polytechnic State University, \\ San Luis Obispo
}

\author{
In Partial Fulfillment \\ of the Requirements for the Degree
}

Master of Science in Agriculture with Specialization in Animal Science

by

Jane Marie Isquith

June, 2013 
(C) 2013

Jane Marie Isquith

ALL RIGHTS RESERVED

Page ii 
TITLE:

AUTHOR:

DATE SUBMITTED:

COMMITTEE CHAIR:

COMMITTEE MEMBER:

COMMITTEE MEMBER:
Establishment and validation of a novel Fucci (fluorescent ubiquitination-based cell cycle indicator) in an in vitro chronic myeloid leukemia model

Jane Isquith

June, 2013

Matt Burd, D.V.M., M.S., Professor

Animal Science Department

California Polytechnic State University, San Luis Obispo

Elena Keeling, Ph.D., Professor

Biology Department

California Polytechnic State University, San Luis Obispo

Leslie Crews, Ph.D., Project Scientist University of California, San Diego

Sanford Consortium for Regenerative Medicine 


\begin{abstract}
Establishment and validation of a novel Fucci (fluorescent ubiquitination-based cell cycle indicator) in an in vitro chronic myeloid leukemia model

Jane Marie Isquith

The deregulation of the cell cycle is important in the leukemic transformation and in the continued discovery of more efficient targeted therapies. After elucidation of the role of the RNA editor ADAR1 in the malignant reprogramming of Chronic Myeloid Leukemia (CML) progenitors to leukemia stem cells (LSCs) during the chronic phase to blast crisis stages of the disease, current research seeks to uncover the mechanism of action of ADAR1 in this process. To study the potential role ADAR1 plays in the changes to cell cycle during this disease progression, a novel Fucci (fluorescent ubiquitination-based cell cycle indicator) system was introduced into the CML cell line K562 through stable transduction of both Fucci vectors. This allows for the visualization of the cell cycle via FACS (fluorescence activated cell sorting), and fluorescent live imaging. This system was validated for use in therapeutic CML research through preliminary evaluation of ADAR1 activity in specific cell cycle stages as well as the effects of a potentially novel CML therapy, 8-azaadenosine, on the cell cycle. Completion of this work shows successful creation of a novel generation of Fucci cell line; which will aid in the clarification of the changes which occur in the cell cycle during CML disease progression, as well as the effects of CML targeted therapies on cell cycle regulation though quantitative and qualitative analysis.
\end{abstract}




\section{ACKNOWLEDGMENTS}

I would like to thank my committee chair, Dr. Matt Burd, for all his years as my faithful advisor; being there to fight the system with me, and who motivated me to always keep striving for greater things. I am also grateful to my committee, Dr. Elena Keeling and Dr. Leslie Crews, whose scientific prowess shaped this project into something I am very proud of. To Dr. Catriona Jamieson and the Jamieson Lab at UCSD, thank you all for welcoming me into the lab and for the continuous support; it was an unbelievable opportunity to work with so many smart, talented, and wonderful people. And to Dr. Fay Jiang, it was a pleasure being your mentee, I could not have asked for a better team to work with. 


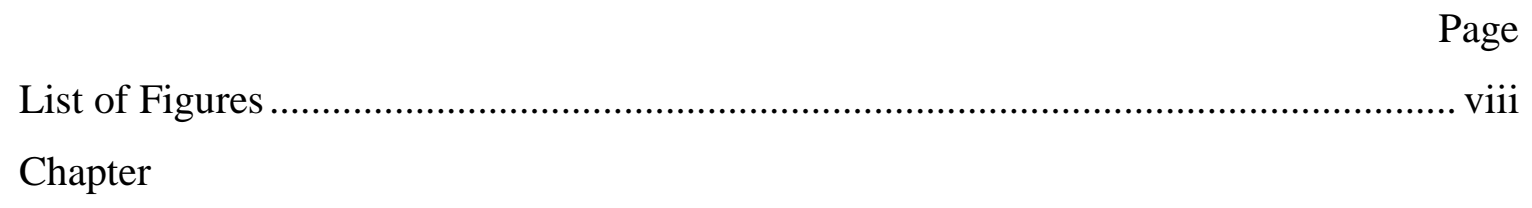

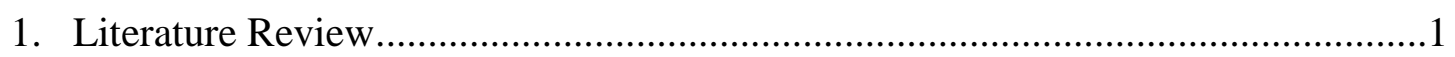

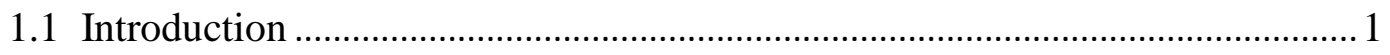

1.2 Acute and Chronic Myeloid Leukemia.......................................................2

1.3 Cancer Stem Cells .............................................................................

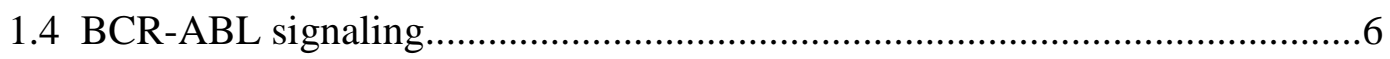

1.5 Current Treatments: Tyrosine Kinase Inhibitors ......................................8

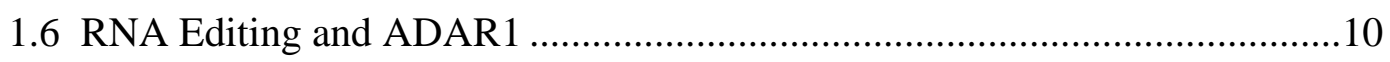

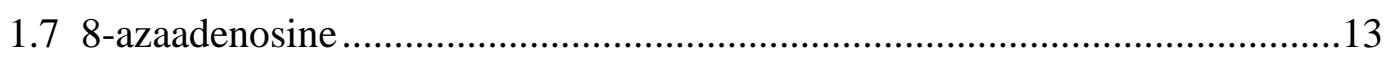

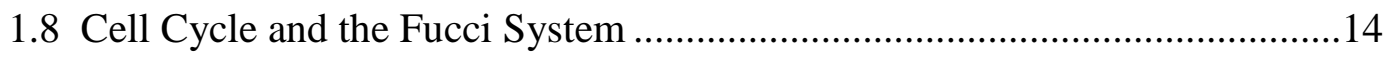

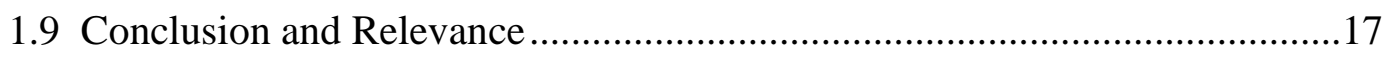

2. Establishment and validation of a novel Fucci (fluorescent ubiquitination-based cell cycle indicator in an in vitro chronic myeloid leukemia model.....................18

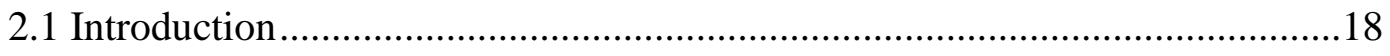

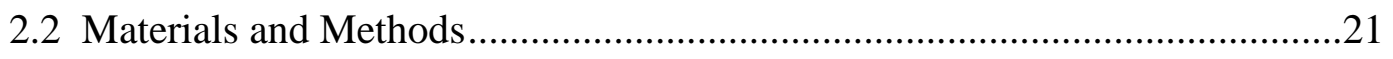

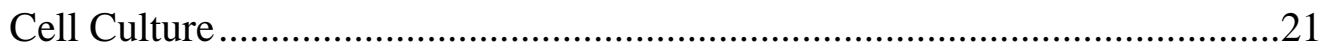

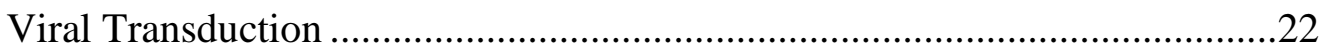

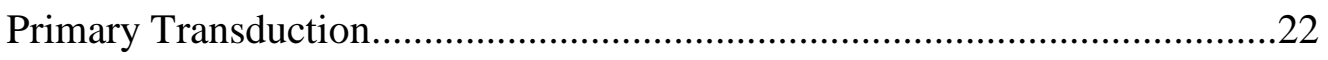

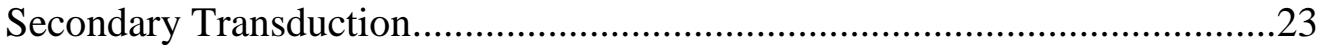

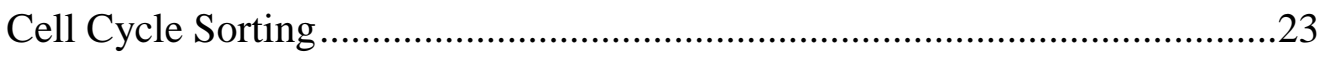

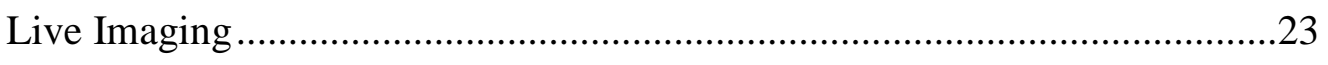

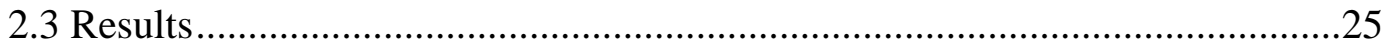

Stable Transduction of Fucci-R and Fucci-M..........................................25

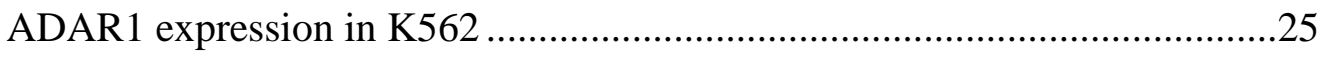

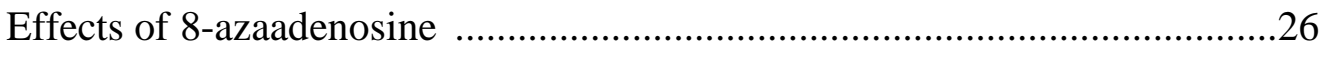


2.4 Discussion

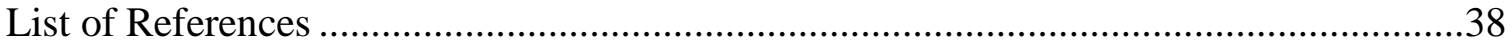

Page vii 


\section{LIST OF FIGURES}

Figure Page

1. Establishment of a stably double positive Fucci R-M K562 cell line.....................31

2. Analysis of the cell cycle using the Fucci system .............................................33

3. ADAR1 expression in specific stages of the cell cycle ......................................34

4. Effects of 8-azaadenosine titration on cell cycle .................................................35 


\section{CHAPTER 1}

Literature Review

\subsection{Introduction}

The study of cancer stem cells (CSC) and their role in the disease transformation and progression is at the forefront of research being conducted in the field of leukemia. According to the Centers for Disease Control and Prevention, cancer is the second leading cause of death in the United States. Leukemia is defined as a cancer of the blood or bone marrow and is categorized by four major subsets, distinguished by the affected lineage and severity: acute myeloid leukemia (AML), acute lymphocytic leukemia (ALL), chronic lymphocytic leukemia (CLL), and chronic myeloid leukemia (CML). The American Cancer Society report that CLL constitutes about one third of leukemia diagnoses. While the other manifestations of leukemia affect older people, ALL is most prevalent in children. There will be approximately 6.000 new cases of ALL in 2013, about one third affecting adults. CML accounts for approximately $10 \%$ of all new leukemia diagnoses, and it is estimated that there will be almost 6,000 new diagnoses in 2013. New cases of AML are estimated to be approximately 14,500. 


\subsection{Acute and Chronic Myeloid Leukemia}

While there exist similarities between AML and CML, the disease state and subsequent malignancy is driven through different molecular events. These differences are key to understanding the future of targeted therapies. AML can be seen as the more severe, end-stage prognosis, whereas CML undergoes a potentially slower progression through three stages: chronic phase, accelerated phase and blast crisis [1]. CML has also been described as biphasic, in which chronic phase and blast crisis are the two manifestations of the disease [2]. The malignancies of each stage of CML differ slightly, and it is crucial to note that blast crisis CML possesses great similarities to AML [3]. AML phenotypically presents as an excess of immature hematopoietic cells, known as leukemic blasts, which do not possess the characteristics of a cell type likely to allow for metastasis of the disease [4]. This finding gave rise to the work toward elucidating the population of cells responsible for propagation of the cancerous cells [4]. Unlike AML, CML undergoes a progression of the disease from chronic phase $(\mathrm{CP})$ to the terminal blast crisis (BC) stage. Blast crisis closely resembles AML, with an accumulation of immature blasts with enhanced self-renewal capacity and worsening prognosis $[5,6]$. After progression from $\mathrm{CP}$ to $\mathrm{BC}$, life expectancy drops dramatically to approximately 6 months [7]. CML was the first cancer to be defined at the molecular level when the reciprocal translocation of chromosome 9 and 22 was identified, which results in the generation of the Philadelphia chromosome $\left(\mathrm{Ph}^{+}\right)$. This chromosomal abnormality was first discovered and studied in leukemia in 1960 by Nowell and colleagues, and further characterized into the next decade $[8,9]$. Upon translocation there is the creation of the 
$\mathrm{BCR} / \mathrm{ABL}$ tyrosine kinase fusion protein $[5,10,11]$. This $210 \mathrm{kD}$ oncogenic fusion protein, which is also referred to as $\mathrm{P} 210^{\mathrm{bcr} / \mathrm{abl}}$, leads to unregulated activity of the tyrosine kinase signaling cascade, and effects such as unchecked proliferation [11]. Based on the work examining the molecular pathogenesis of the leukemic transformation, it seems evident that this translocation can occur at the level of one hematopoietic stem cell (HSC); and this now malignant progenitor undergoes clonal expansion to facilitate the aberrant leukemic phenotype $[11,12]$. 


\subsection{Cancer Stem Cells}

Elucidation of the cancer stem cell (CSC) population in leukemia was first studied in AML by John Dick and colleagues in the 1990's. Through these experiments, a definition for CSCs in AML was established characterizing the population of cells in AML which are capable of maintenance and metastasis of leukemia. As in normal stem cells, these cells possess the ability to self-renew and differentiate, allowing propagation of the disease as demonstrated by in vivo studies in which both primary and secondary transplantation of the hypothesized CSC population isolated from human AML patients yielded the same AML disease state [4]. Subpopulations of leukemic cells can be identified by their expression of cell surface proteins. The two markers now commonly used for the identification of leukemia stem and progenitor populations are the surface proteins CD34 and CD38. While the functions of these proteins are still somewhat elusive, it is known that CD34 plays a role in cell adhesion as well as hematopoietic stem cell (HSC) trafficking [13]. While CD $34^{+}$cells are known to have the ability to reconstitute the hematopoietic system in patients undergoing bone marrow transplantation [14]. CD38 is also a transmembrane glycoprotein, which also belongs to a family of cytosolic and membrane bound enzymes that catalyze nicotinamide adenine dinucleotide (NAD) reactions $[15,16]$. It has also been shown to function in calcium mobilization and signaling [17]. In both of these manifestations of leukemia, the CSC population is known to be $\mathrm{CD} 34^{+} \mathrm{CD} 38^{-}$, in comparison with the progenitor population, which are known to have double positive expression of these markers, therefore making them $\mathrm{CD} 34^{+} 38^{+}$. These two populations are distinct based on the capacity of the 
$\mathrm{CD}^{+} 4^{+} \mathrm{CD} 38^{-}$population to self-renew, whereas in $\mathrm{AML}$ the $\mathrm{CD} 34^{+} \mathrm{CD} 38^{+}$population does not [7]. The method of identifying the potential for malignant populations of cancerous cells to self-renew is demonstrated through the transplantation of cell populations identified by their marker expression in mouse models. Cell populations that are able to engraft into host animals and initiate disease are in that way validated as being capable of potentiating the disease $[18,19]$. These cells can also be identified in vitro nature through colony assay studies involving the re-plating of cells in culture to classify self-renewal [20]. 


\subsection{BCR-ABL signaling}

Upon translocation, creation of the $B C R-A B L 1$ oncogene results in translation of the Bcr-Abl fusion protein, which resides solely in the cytoplasm, as compared to normal Abl which is found in both the nucleus and cytoplasm. This translocation also causes the constitutive activation of the Abl tyrosine kinase and subsequent deregulation and oncogenic activity of this signaling pathway [21]. This aberrant signaling is manifested through the apposition of the $\mathrm{Bcr}$ and $\mathrm{Abl}$ protein, causing constitutive activation of $\mathrm{Abl}$ signaling through dimerization and tetramerization and subsequent activation of downstream signaling [21-23]. Signaling activated or promoted by Abl activation includes PKC, RAC, RAS, PI3K and Jak cascades [24]. Very early studies in CML showed that the origins of the disease can begin at the level of a single hematopoietic cell $[25,26]$. The ensuing expansion of the leukemic clone is hypothesized to lead to progression of the disease, however new research is showing there may be multiple other factors involved in regulation and advancement of the disease [5, 11, 27]. CML is characterized phenotypically by the rapid growth and expansion of a specific subset of $\mathrm{CD} 4^{+} \mathrm{CD} 38^{+}$hematopoietic progenitor population known as the granulocyte macrophage progenitor (GMP) population $\left(\mathrm{CD}_{4} \mathrm{RA} \mathrm{A}^{+} \mathrm{CD}_{123}{ }^{+}\right)$, which leads to an unchecked accumulation of other myeloid cells [28]. These myeloid progenitor cells leave the bone marrow niche prematurely, and subsequently lead to the accumulation in the blood, along with an increased number of mature cells as well $[2,11]$. In comparison to AML in which there is unchecked growth and expansion of immature cells, CML chronic phase $(\mathrm{CP})$ exhibits abnormal numbers of more mature cells $[5,7]$. In 
concurrence with most other forms of cancer, CML expansion is characterized by unregulated proliferation and differentiation, as well as changes in the quiescent population [10]. This expression pattern resembles that of normal stem cells, leading to the hypothesis that there may be factors driving these propagating cells towards malignancy. Blast crisis CML is driven by the leukemia stem cell (LSC) population, which manifests phenotypically as overexpansion of an abnormal GMP population. These leukemic cells now possess an uncharacteristic ability for self-renewal, while exhibiting a less mature phenotype, as seen by the massive accumulation of these immature progenitor cells [7, 29]. 


\subsection{Current Treatments: Tyrosine Kinase Inhibitors}

Current treatments of CML target the effects of the BCR-ABL fusion oncoprotein with tyrosine kinase inhibitors (TKI) including imatinib and dasatinib, which mechanistically function to inhibit the deleterious and oncogenic downstream effects of this unregulated kinase pathway. While this targeted therapy has shown success in patients with CML CP, many patients undergoing treatment with these drugs will initially exhibit or develop a clinical resistance [30,31]. Abl kinase inhibitors have been shown to inhibit the in vitro effects of unchecked kinase activity such as cellular proliferation, as well as in vivo dose dependent inhibition of tumor growth [32]. These kinase inhibitors function as a competitive inhibitor to the ATP binding site of the Abl kinase [1]. Imatinib prevents $\mathrm{BCR}-\mathrm{ABL}$ activity by binding the inactive conformation and prohibiting the transition into the active form $[6,33]$. Also important to this breakthrough therapy is its selective nature as demonstrated by its ability to target activity of only Bcr-Abl expressing cells. In addition it does not show deleterious effects on normal hematopoiesis [1,32]. Imatinib (also known as Gleevec or STI571) was the first generation Bcr-Abl kinase inhibitor, shown to function to achieve a complete cytogenic response (CCR); a state defined as eradication of $\mathrm{Ph}^{+}$cells in the bone marrow [7]. Despite this success via treatment with first generation TKIs such as imatinib, some patients exhibit a primary resistance, in which there is no initial cytogenic response to treatment; or a secondary resistance in which patients lose their initial response to imatinib. The most common and well defined cause of this resistance is due to $B C R-A B L$ mutations. Numerous amino acid mutations affecting the kinase domain of the protein have been characterized, leading to 
the inability of the drug to bind [6,34]. It is also hypothesized that this may be in part due to the reliance on the CSC and progenitor population in the progression of CML, although the mechanism by which quiescent LSC remain resistant to treatment is not fully defined [10]. Studies have shown that the quiescent $C D 34^{+}$population can survive through treatment of imatinib, whereas the most affected population of cells are those that are dividing [35]. Dasatinib, the second generation SRC/BCR-ABL kinase inhibitor, has been shown to be more potent than imatinib, however resistance can still be developed, and after treatment, there seems to be minimal reduction in the quiescent population [30, 36]. Dasatanib has a slightly different mechanism of action than its predecessor; this 325 fold more potent TKI binds a similar region of the ATP binding domain in the active conformation of BCR-ABL $[6,37]$. The CSC or progenitor population of cells may be resistant to these targeted therapies and therefore contribute to the reemergence of the disease after treatment, as indicated by the ability to achieve a CCR through imatinib treatment followed by the reemergence of the disease [38]. Studies conducted by the Jamieson lab are looking into possible explanations for what drives the growth and maintenance of these CSCs in order to find advanced therapies for more effective prediction and prevention of CML progression. 


\subsection{RNA Editing and ADARI}

The role of the CSC and progenitor populations in CML is crucial to understanding the mechanisms for propagation and potential therapeutic targets. The exposition of the mechanisms maintaining the stem-like qualities of these cells is at the forefront of CML research. Besides the ability for self-renewal and differentiation, there seems to be deregulation of the cell cycle, and an exaggerated quiescence in these stem and progenitor cells $[10,30]$. Studies have been conducted examining the mechanisms of action by which cells acquire these new found characteristics ideal for the promotion of metastasis. The missplicing of GSK3 $\beta$ has been shown to distinguish BC from CP CML [39]. Aberrant splicing can also lead to the generation of oncoproteins, including generation of novel protein isoforms which can be tumorigenic or play a role in the development of therapeutic resistance [40]. The identification of these abnormalities in RNA processing has generated much interest in the role of RNA regulatory pathways in cancer initiation and progression. One mechanism responsible for this promotion of a malignant progenitor through RNA modification may be the effects of RNA editing specifically by the adenosine deaminase acting on double stranded RNA (ADAR) editases. RNA editing is classically defined as the change in coding properties of RNA, through the modification of nucleotide sequence [41, 42]. ADAR was first identified in Xenopus laevis in 1987 and was subsequently studied in other organisms [43] There are three forms of ADAR in mammals (ADAR1, ADAR2, and ADAR3); however ADAR1 is the most highly characterized in hematopoietic cell development and this form is the focus of studies in our lab. ADAR1 is present in two isoforms; a $150 \mathrm{kDa}$ interferon 
inducible protein (p150) that is found in both the nucleus and the cytoplasm, as well as a truncated $110 \mathrm{kDa}$ protein $(\mathrm{p} 110)$ that localizes solely to the nucleus $[44,45]$. ADAR1 has been shown to edit RNA extensively in primate-specific Alu sequences through adenosine-to-inosine (A-to-I) editing with a variety of post-transcriptional changes. Alu elements are non-coding retrotransposons that account for more than $10 \%$ of the human genome [46]. The A-to-I change in nucleotide sequence is recognized functionally as an A-to-G change [47]. Results of this editing include creation of alternative splicing sites $[44,48]$ as well as changes to RNA structure [42, 49]. Alternative splicing can create multiple protein transcripts from a single gene, which has already been indicated in cancer biology through the generation of novel oncogenic isoforms; as is the case with the missplicing of GSK3 $\beta$. RNA editing by ADARs on Alu sequences has also been shown to occur at high frequency during embryonic development [50]. ADAR1 has also been linked specifically to the survival of hematopoietic progenitor cells [51, 52]. In this work, hematopoietic progenitor cells only propagated and survived to be found in bone marrow and peripheral blood when knockdown of the Adar allele was not induced. This logically leads to the supposition that ADAR editing may play a crucial role in cancer, and specifically in CML. This hypothesis is supported by findings of increased ADAR1 expression in other forms of cancer, including astrocytomas [53]. The latest work done in the Jamieson lab by Jiang, et al (PNAS, 2013) has linked ADAR1 p150 expression to CML disease progression and the malignant transformation of progenitors to leukemia stem cell (LSC) like cells. This works shows increased ADAR1 p150 in CP CML compared to normal cord blood and a subsequent significant increase as the disease progresses to BC. ADAR1 is also known to have distinct effects on the cell cycle; 
specifically overexpression of ADAR1 leads to the acceleration of the cell cycle as well as the proportional decrease of cells in G0 and G1 cells paired with an increase in S phase cells. The proposed mechanism of this accelerated cell cycle is through the upregulation and activity of cyclin dependent kinase 2 and it's corresponding cyclin A, which naturally accumulates in S phase of the cell cycle. [54]. Therefore, elucidation of the changes that occur in the cell cycle of CML cells, both in general and due to the effects of ADAR1, is crucial to furthering the understanding of CML initiation and progression. 


\subsection{8-azaadenosine}

After elucidation that ADAR1 does in fact play a role in the blastic transformation [55], research can be focused on examining the numerous possible mechanisms of action through which ADAR1 could function. 8-azaadenosine is a compound that can be chemically synthesized and has been shown to be a substrate for adenosine deamination by ADAR2 $[41,56]$. ADAR2 resides in the same family of ADAR enzymes as ADAR1, and have similar editing activity, however there are some differences in editing site preference $[57,58]$. 8-azaadenosine has been shown to incorporate into polynucleotides as 8-azaadenine and 8-azaguanine [59]. 8-azaadenosine has also been studied in cancer research, and examined as an anti-tumor agent in human colon carcinoma cell lines [60]. It has also been proposed to also cause cell death through inhibition of DNA synthesis [59], so future studies will be necessary to distinguish between effects of this inhibitor on RNA editing and DNA alterations. Based on these factors, work with 8-azaadenosine in CML will seek to elucidate the effects of 8-azaadenosine on ADAR1 activity and cell cycle regulation in CML. 


\subsection{Cell Cycle and the Fucci System}

While it has been a common technique to examine cell proliferation using cell tracking dyes such as carboxy-fluorescein diacetate succinimidyl ester (CFSE) that labels parent cells but becomes diluted upon generation of daughter cells. There are, however, alternative methods to investigate not only the general proliferation activity but also to study the distinct phases of the cell cycle. This is of importance in the field of CML research due to the fact that there is most like aberrant manifestations of the cell cycle in cancerous cells. The Fucci system (fluorescent ubiquitination-based cell cycle indicator) is an emerging tool that can be used to examine the cell cycle in numerous applications. This system exploits two distinct proteins that are ubiquitinated at different stages of the cell cycle by fusing them with either green or red fluorescent tags [61]. This system was set up after previous research illuminated the oscillating ubiquitination by E3 ubiquitin ligases controlling the different transitions throughout the cell cycle [62]. The fluctuation

of these ligases throughout the cell cycle regulates the degradation of proteins that control transitions from one stage of the cell cycle to the next. Cyclin-dependent kinases (CDK) regulate these transitions and are active dependent on the accumulation of stage specific cyclins which facilitate entry into a specific stage, or alternatively inhibit exit from a stage $[63,64]$. This requires changes in cyclin levels throughout the cell cycle, so that there is a logical progression through each stage. Cyclins, and other proteins involved in the regulation of the cell cycle, are marked for degradation through ubiquitination [64]. There are three components that work in conjunction to mark proteins for degradation via ubiquitin mediated proteolysis, E1 ubiquitin activating enzymes, E2 ubiquitin 
conjugating enzymes, and E3 ubiquitin ligases; E3 ubiquitin ligases being the enzyme that is responsible for the covalent attachment of ubiquitin to the protein of interest [65]. There are two prevalent E3 ubiquitin ligases, anaphase promoting complex (APC) and SKP1-CUL1-F-box-protein complex (SCF), function through feedback loops to show reciprocal activity during late $\mathrm{M} / \mathrm{G} 1$ and S/G2 phases, respectively [64]. In the original generation of these probes, a direct substrate of $\mathrm{SCF}^{\mathrm{skp} 2}$, $\mathrm{Cdt} 1$, was fused to $\mathrm{mKO} 2$ (monomeric kusabira orange 2). Since $\mathrm{SCF}^{\mathrm{skp} 2}$ is the $\mathrm{E} 3$ ubiquitin ligase that polyubiquitinates Cdt1, which marks it for degradation, Cdt1 shows accumulation (and subsequent red fluorescence) during the G1 phase of the cell cycle. Geminin, which is ubiquitinated by $\mathrm{APC}^{\mathrm{cdh} 1}$, was similarly fused to $\mathrm{mAG}$ (monomeric Azami Green) leading to accumulation of green fluorescence in $\mathrm{S} / \mathrm{G} 2 / \mathrm{M}$, as $\mathrm{APC}^{\mathrm{cdh} 1}$ is active during $\mathrm{G} 1$. This progression of the ubiquitination linked fluorescence therefore also exhibits yellow fluorescence during the G1/S transition, as Cdt1 accumulates during G1 and the geminin begins to no longer be degraded in S phase, there is an overlap in the presence of both proteins [61]. and is colorless when cells are in G0 [61]. In the second generation of Fucci vectors, mCherry (which displays red fluorescence) was fused to Cdt1 and mVenus (which displays green fluorescence) was fused to Geminin to allow for a sharper color contrast between cell cycle phases. Upon cell death, the cell membranes become permeable, and as these vectors are not tightly bound, fluorescence is lost through the compromised membranes [66]. In the experiments describing the generation of these second generation constructs, lentivirus was created through co-transfection of the vectors encoding either mCherry-hCdt 1 or mVenus-hGem and then transduced into $\mathrm{HeLa}$ or NMuMG cell lines and selected via single cell cloning or FACS. In the experiments 
conducted in the Jamieson lab, we employed a similar technique of lentiviral transduction using two different vectors; one encoding mCherry-hCdt 1 and the other mVenus-hGem and sorted for stable transduction by FACS. These second generation Fucci lentiviral reporters were used to investigate cell cycle regulation in CML cell lines. These vectors can be introduced into cell lines using lentiviral transduction establishing stable lines which can be used to elucidate the changes in the cell cycle in progressive stages of CML as well as the effects of current and novel therapies on the cell cycle. Examining how CML therapies modify the cell cycle is crucial to the development of combination therapies to prevent the emergence of therapeutic resistance. The development of the Fucci system in CML models will allow for advances in targeted therapy of CML. This system can be used to sort populations of cells in different phases of the cell cycle in order to study the effects of novel treatments. 


\subsection{Conclusion and Relevance}

Cumulative evidence demonstrates a role for abnormal ADAR1-mediated RNA editing and cell cycle deregulation [67] in CML progression, underscoring the need for more in-depth analysis of these processes and the connection between aberrant RNA editing and cell cycle control in CSC and other malignant cell populations. The elegant design of the Fucci vector system allows for in vitro analysis of these processes using CML cell lines. In this context, the main hypothesis of this project is to be able to investigate the changes in cell cycle regulation in CML initiation and progression through introduction of a Fucci system into a CML cell line. Thus, the overall goal of this project was to develop an optimized protocol for transducing cells with multiple Fucci vectors in order to visualize and quantify the cell cycle. The use of splicing and adenosine deaminase inhibitors in combination with current CML therapies is currently under investigation for use as potential CML therapies. There is substantial interest concerning the effects of these potential treatments on the cell cycle. It remains a crucial element of CML research to identify if there is a therapy that can target the quiescent cell population or conversely a factor that can drive those quiescent cells into cycling normally so that current treatments will have a more profound and lasting effect. The secondary objective of this project was to validate the effects of potential anti-CSC therapies in a Fucci CML cell line model using a putative ADAR1 inhibitor- 8-azaadenosine, on the cell cycle regulation in a CML. 


\section{CHAPTER 2}

\section{ESTABLISHMENT AND VALIDATION OF A NOVEL FUCCI (FLUORESCENT UBIQUITINATION-BASED CELL CYCLE INDICATOR) IN AN IN VITRO CHRONIC MYELOID LEUKEMIA MODEL}

\subsection{Introduction}

Despite advances in recent decades that have introduced molecularly-targeted therapies for chronic myeloid leukemia (CML), the molecular events driving leukemic transformation and disease progression of CML remain poorly-defined and require further elucidation for advances to be made in translational medicine. CML is classically defined by the expression of the BCR/ABL fusion oncoprotein caused by the reciprocal translocation of chromosome 9 and 22, resulting in the formation of the Philadelphia chromosome $[5,10,11]$. This translocation and oncoprotein generation causes the constitutive activation of the Abl tyrosine kinase and subsequent deregulation and oncogenic activity of this signaling pathway [21]. The ensuing expansion of the leukemic clone possessing the BCR/ABL mutation is hypothesized to lead to progression of the disease, however new research is showing there may be other factors involved in regulation and advancement of the disease. These include additional mutations such as in the Jak/STAT signaling cascade, specifically in $J A K 2$, as well as subsequent triggers of cellular deregulation $[5,11,27,68,69]$.

Previous research recently published by the Jamieson lab has shown that the RNA editing enzyme ADAR1 (adenosine deaminase acting on RNA-1) is involved in the progression of CML from chronic phase (CP) to blast crisis (BC). Expression of one specific inflammation-responsive ADAR1 isoform, ADAR1 p150, has been linked to CML disease progression and the malignant transformation of progenitors to leukemia stem cell (LSC) like cells [55]. ADAR1 is known to edit primarily Alu repeat sequences through adenosine-to-inosine (A-to-I) editing. The numerous downstream effects of this editing include changes in cell proliferation and differentiation [54]. 
Changes in the cell cycle are known to occur during the leukemic transformation as well as disease progression in CML. There has been great interest pertaining to the deregulation of the cell cycle and the quiescent population of leukemic stem cells (LSCs). LSCs have been indicated in being at least partially responsible for the transformation of CML from CP to BC; notably due to the inherent quiescent subpopulation of LSC. This population is able to propagate the disease through asymmetric division leading to the production of a quiescent daughter cell as well as one able to differentiate into a mature cancerous cell [70]. LSCs and the quiescent population of cells in any cancer, including CML, play a key role in development of therapeutic resistance, because they are often not affected by current anti-cancer treatments [10]. After the elucidation of ADAR1 activity in CML disease progression, the specific roles of ADAR1 can be examined. We carried out a series of experiments in leukemic cells engineered to express high levels of ADAR1 p150 to further investigate the role of ADAR1 in leukemic progression. We observed qualitative changes in cells overexpressing ADAR1 in both a CML cell line, K562, and corresponding alterations in primary CML patient samples transduced with a lentiviral vector overexpressing ADAR1 p150. These changes include cell size as well as cell proliferation. Based on these findings we hypothesize that increased ADAR1 expression in CML affects the cell cycle potentially being one of the mechanisms by which ADAR1 modulates blastic transformation of CML.

To study the cell cycle in CML, a current commonly used method employs immunostaining and flow cytometry for cell cycle analysis. However, visualization is limited to assessment of cell surface protein marker expression. In this study, we show a novel system that allows for visualization and isolation of cells at individual phases of the cell cycle, and creates a method in which to study in vitro the effects of CML therapies on cell cycle.

The Fucci system (fluorescent ubiquitination-based cell cycle indicator) is an emerging tool that can be used to examine the cell cycle in numerous applications by using two complexes that have ubiquitination activity at different stages of the cell cycle and fusing them with either green or red fluorescent tags [61]. This system has been 
shown to be effective in studying changes in the cell cycle caused by anticancer drugs in normal cell lines [61], but has yet to be studied in an in vitro CML model. In this work, the establishment of the Fucci system in the CML cell line K562 is validated through FACS analysis, confocal imaging, as well as preliminary tests of a novel therapeutic agent and putative ADAR1 inhibitor, 8-azaadenosine. 


\subsection{Materials and Methods}

\section{Cell Culture}

All cell lines were maintained at $37^{\circ} \mathrm{C}, 5 \% \mathrm{CO}_{2}$ and supplemented with corresponding complete media as per product recommendations. K562, LAMA84, LAMA87 and KU812 cell lines were purchased from ATCC (Manassas, VA). K562 were grown in DMEM supplemented with 10\% FBS (Gemini Bio-Products, Sacramento, CA), $1 \%$ Glutamax (Life Technologies, Carlsbad, CA), and 1\% NEAA. LAMA84 and KU812 were grown in RPMI 1640 (Life Technologies, Carlsbad, CA) supplemented with 10\% FBS. LAMA87 were grown in RPMI 1640 with 20\% FBS supplementation. Cells were maintained at approximately $70-80 \%$ confluence. For multi area time lapse images, complete media was supplemented with 1:100 penicillin/streptomycin. In some experiments, a stromal co-culture system was used to more closely emulate the normal bone marrow environment (Figure 2). Murine SL and M2 cell lines were maintained in culture separately until use in co-culture experiments. SL cells were grown in DMEM supplemented with 10\% FBS, 1\% L-glutamine, 1\% NEAA and 1:100 penicillin/streptomycin. M2 cells were grown in RPMI supplemented with 10\% FBS, $1 \%$ L-glutamine, and 1:100 penicillin/streptomycin. Both cell lines were split approximately once per week using $0.25 \%$ trypsin; SL cells were split 1:10, M2 cells were split 1:20. Every five passages, transgenic clones were selected by culturing for one passage in $1 \mathrm{X}$ G418 and 1X Hygromycin.(Life Technologies, Carlsbad, CA). One day before coculture, cells were trypinized and collected in a $50 \mathrm{~mL}$ tube, washed with complete media, and resuspended in $1 \mathrm{X}$ mitomycin-C $(0.01 \mathrm{mg} / \mathrm{mL})$ and incubated for 3 hours at $37^{\circ} \mathrm{C}$. After incubation cells were washed with PBS and plated 1:1. After 24 hours, SL/M2 media was aspirated and K562 cells were added in corresponding media. 


\section{Viral Transduction}

\section{Primary Transduction}

To increase transduction efficiency and initial cell survival, cells were transduced with the two vectors independently. After creation of a stable Fucci-R-transduced population, Fucci-M transduction was performed on this single positive population. All cell lines were transduced first with lentiviral Fucci-R expressing mCherry. A $100 \mu 1$ cell suspension was plated into 10 wells of a 96 -well plate. Concentrated Fucci-R virus (made by the UCSD Sanford Consortium for Regenerative Medicine viral vector core) was diluted in each line's corresponding media and $5 \mu$ l of diluted virus was added to each well. Cells were then incubated for 3-5 days and mCherry expression was monitored by fluorescence microcopy.

After 3-5 day incubation with the virus in 96 well plates, cells were collected and transferred into 2 wells of a 6 well plate per condition. These were expanded 1-2 days then transferred into a T75 flask for continued propagation. Cells were expanded until reaching a desired volume for sorting, usually $\geq$ one T75 flask. At the time of sorting, cells were collected by centrifugation, washed with HBSS plus $2 \%$ FBS and then sorted for mCherry expression using a BD FACSAriaII. Only live cells were selected by resuspending cells in a 1:1000 pI solution and gated for pI negative cells only. Cells with high red fluorescence intensity were sorted into $1 \mathrm{~mL}$ FBS with $20 \%$ penicillin/streptomycin and kept on ice until transfer into warm complete media. To increase survival, this primarily sorted population of cells were plated at a density of $>30 \%$ confluence in a corresponding volume container. In most cases, after primary sorting, cells were plated into a 6-well plate for initial expansion. Once cells reached approximately $80 \%$ confluence they can be transferred to larger plates or flasks for expansion. 


\section{Secondary Transduction}

After expansion of Fucci-R positive cells, $100 \mu \mathrm{l}$ of cells were plated into 10 wells of a 96 well plate. Concentrated Fucci-M lentivirus (supplied by the UCSD Sanford Consortium for Regenerative Medicine viral vector core) was diluted with RPMI 1640 supplemented with $20 \%$ FBS and $5 \mu$ l diluted virus was aliquoted to each well. Cells were incubated for 3 days before collected and transferred to a 6-well plate. Cells were maintained in culture and expanded until reaching an appropriate confluence to sort. Live cells were sorted for either mVenus expression or expression of both mCherry and mVenus, which presented at a yellow emission caused by the overlap of the two fluorophores. This population was then expanded for subsequent experiments. Stable transduction was monitored by fluorescence expression.

\section{Cell Cycle Sorting}

After development of the stably transduced K562-Fucci cell line, cells were sorted based on fluorescence determined by the Fucci system on the BD FACSAriaII using DAPI as a live/dead indicator. Colorless cells were sorted as cells in G0; Fucci R positive cells (mCherry only) were sorted as G1 cells; Double positive cells expressing both mCherry and mVenus were sorted as cells in the G1/S transition; and cells expressing only FucciM (mVenus only) were sorted as cells in G2. Cells were sorted into $2 \mathrm{mLs}$ FBS with 1:100 penicillin/streptomycin. Cells were then immediately plated in K562 complete media or frozen in $90 \%$ FBS, $10 \%$ DMSO. These were thawed directly into K562 complete media for live cell imaging of synchronized cell populations.

\section{Live Imaging}

Confocal and time lapse images were taken using a computer-assisted Olympus Fluoview FV10i fluorescence microscope (Olympus America, Center Valley, PA). Cells were plated at low densities in normal culture media. Cells were maintained at $37^{\circ} \mathrm{C}, 5 \%$ 
$\mathrm{CO}_{2}$ in the incubation chamber of the microscope for extended imaging. For time lapse imaging, cells were plated into $35 \mathrm{~mm}, 14 \mathrm{~mm}$ microwell with no. 1.5 coverglass $(0.16$ $0.19 \mathrm{~mm}$ ) Poly-d-lysine coated culture dishes (MatTek Corporation, Ashland, MA). Images were taken at 5 minute intervals, for varied amounts of time no less than 24 hours to ensure documentation of cell division. 


\subsection{Results}

\section{Stable transduction of Fucci-R and Fucci-M}

The CML cell line K562 was successfully stably transduced with both Fucci-R and Fucci-M lentiviral vectors, and has maintained expression of the two vectors for greater than 20 passages. Stable transduction was assessed by FACS sorting based on fluorescence; followed by regular monitoring with a fluorescence microscope (Figure 1). Over numerous subcultures, no loss of fluorescence activity was detected. The other cell lines, LAMA 84, LAMA 87, and KU 812 were successfully transduced with a single vector but due to the stringent selection process ensuring that all selected cells have been transduced, they were never expanded to the density required for secondary transduction (results not shown). Future work can be performed to create double positive stable transduction in these cell lines.

\section{ADAR1 expression in K562}

K562 Fucci R-M cells were sorted on a BD FACSAria II to isolate single populations of cells in different stages of the cell cycle using the Fucci system (Figure 2). The populations of interest that we are able to sort are G0, which are colorless; G1, which express only mCherry; cells in the G1/S transition during which time both markers are expressed, leading to an overlap in the two fluorophores; and also G2/M, in which there is solely mVenus expression. This technique presents not only a method of sorting cell stages of interest, but it also allows for synchronization of cell populations. This is especially useful in cell lines where this presents a challenge as other techniques such as nutrient starvation is not sufficient for synchronization, as seen in our own experiments. After sorting, we were able to extract RNA and perform qPCR assessing the expression of ADAR1 in each specific population. These preliminary findings showed no distinguishable differences in ADAR1 isoform expression in any stage of the cell cycle, but did allow us to show that we can in fact analyze specific cell stages for expression of markers of interest (Figure 3). 


\section{Effects of 8-azaadenosine on K562}

To further validate the K562 Fucci system for use with CML therapies, as well as to test the effects of a potential therapeutic agent, we examined the effects of 8azaadenosine on the CML cell line K562 both by FACS analysis as well as through longterm, multi area live imaging (Figure 4). A titration of varying concentrations of 8azaadenosine was performed on K562 Fucci cells in normal culture conditions. After 5 days of treatment, cells were analyzed on the FACSAriaII. We sought to identify which concentration of 8-azaadenosine caused cell death or proliferation, as well whether 8azaadenosine was responsible for any other alterations in the cell cycle, such as a shift or arrest of cells into a particular cell cycle stage. The results of this experiment indicate that as compared to the vehicle (DMSO) only control, low levels of 8-azaaddenosine ( $\leq$ $100 \mathrm{nM}$ ) stimulate an apparent increase in cell numbers, however a large population of the cells remain in G0, with an normal comparative distribution of cells in the other cell cycle stages. At the two highest concentrations of 8 -azaadenosine (1,000nM and 10,000nM), there is noticeable cell death accompanied by a shift in the proportion of cells in each stage of the cell cycle. This is seen as a dramatic decrease in the number of cells in G0, along with an increase in the proportion of cells in the G1/S transition. 


\subsection{Discussion}

In this series of experiments, successful establishment of the Fucci system in the CML cell line K562 has been performed and validated for use in in vitro studies. This novel construction was adapted from the original protocol for use in our CML model. The model provides an important new tool for elucidation of the role of cell cycle modifications in the process of leukemic transformation, as well as the continued efforts to enhance the efficacy of targeted therapies. Although it will be of great relevance to adapt this model into primary patient samples, it was necessary to first optimize the protocol in a well-documented, and clinically relevant cell line. The BCR-ABL+ K562 cell line is used as an in vitro model of CML, and while displays some of the typical drawbacks encountered when using a cell line, it remains useful in preliminary experiments as well as a control to use for comparison to primary CML patient samples. This K562 Fucci line can also be used in preliminary drug screenings, as patient samples are too valuable to use in protocol optimizations such as establishing ideal drug concentrations for use as a possible combination therapy to treat CML.

We were able to establish a stably double positive Fucci R-M K562 cell line and validate it through FACS analysis, long-term confocal live cell imaging, and preliminary drug screening. For these experiments, we began the process of examining ADAR1, a RNA editor which we have previously described as playing a role in the leukemic transformation. One goal of our studies focuses on determining the effects of ADAR1 activity on the cell cycle, and whether deregulation of the cell cycle is a possible mechanism of action by which ADAR1 drives blastic transformation. Previous findings revealed by the Jamieson lab show that ADAR1 overexpression promotes blastic transformation through the reprogramming of progenitor cells into LSCs [55]. Because the cell cycle plays such a crucial role in the fate of LSCs in the maintenance of quiescence, we sought to explore novel methods of studying the changes that occur in the cell cycle during leukemic transformation. In experiments using enforced overexpression of ADAR1 in both K562 as well as primary patient samples, we observed qualitative changes in cellular proliferation and size. This observation, along with other publications 
describing the role of ADAR1 in cell cycle as well as documentation that the cell cycle is deregulated in CML, we extended our studies in elucidating the role of ADAR1 in cell cycle changes in CML. While previous studies have examined the effects of 8azaadenosine in other types of cancer, it has not been evaluated in CML. In these early studies involving 8-azaadenosine, the compound was shown to cause cell death through inhibition of DNA synthesis, and therefore induces antitumor effects $[59,60]$. In corroboration with our findings, we saw that while treatment seems to drive cells into the G1/S transition, they do not successfully divide; which could be explained by the previous research showing 8-azaadenosine inhibits successful DNA synthesis [60]. At the two higher concentrations $(1,000 \mathrm{nM}$ and $10,000 \mathrm{nM})$ of 8 -azaadenosine, there is significant cell death, however it is interesting to note that the surviving cells displayed a noticeable shift into the G1/S transition. There is a lower number of cells in G0 as compared to the control as well as the lower three concentrations of 8-azaadenosine. This suggests that while the cells may be actively cycling, they do not divide or proliferate normally.

Due to the development of clinical resistance in some CML patients after treatment with standard tyrosine kinase inhibitors (TKI), and the likelihood of this resistance leading to the reemergence of the disease in the deadly $\mathrm{BC}$ manifestation, there is ever increasing focus in the entire realm of CML research as well as our lab to develop therapeutic strategies to overcome this resistance. The results of these preliminary experiments suggest that a combination therapy approach may be the most effective; with one agent keeping cells in an active stage of the cell cycle so that another cytotoxic compound could be used to kill the cancerous cells. In clinical CML research there are many therapies that are known to target cells in different stages of the cell cycle, however the need remains for a compound that will drive cells out of G0, as cells in this stage are mostly unaffected by current drugs and therefore primed for development of resistance.

After optimization of in the Fucci system in the K562 cell line, the next steps in this research remain to expand the use of this model system into patient samples, which will allow assessment that more closely models what occurs in vivo. Limitations for the 
use of the Fucci system in primary patient samples include overcoming double positive transduction efficiency in samples with limited cell numbers. We anticipate that only a small fraction of double-positive cells will be able to be isolated, due to the fact that we encounter cell loss after each sort because of the selection process ensuring sufficient transduction. In a cell line, this presented as only a minor setback as these cells will continue to proliferate in almost any condition; however it is known that patient samples do not proliferate nearly as rapidly (if at all) and are often unstable in culture conditions, and cells do not survive if not provided with the right culture conditions. Co-transduction with both vectors at the same time could be considered, however determination of dually transduced cells could be difficult, and again would not entirely overcome the inherent loss of cells during selection.

Continued research is being conducted in our lab to advance the development of the Fucci system for continued use in vitro and in vivo. By cloning in different fluorescent colors, this system can be used in conjunction with overexpression vectors, as well as in in vivo live imaging. We will also continue work with this generation of the K562 Fucci cell line in work examining the role of ADAR1 in cell cycle regulation, as well as other facets of our CML research including testing new therapies, such as a selective Jak2 inhibitor, and investigating the ways in which they modulate the cell cycle. Through continued work with this K562 generation of Fucci vectors, future work includes examining the effects of new and current CML therapies on synchronized populations of cells, such as the effects of 8-azaadenosine on the quiescent G0 population. This will allow us to further examine the effects of quiescence on the development of resistance, as well as develop new strategies for therapies targeting cancerous cells in specific stages of the cell cycle.

In conclusion, this work has resulted in the generation and validation of a novel Fucci vector system in a CML cell line. Further experimentation will allow for the elucidation of the specific changes that occur in the cell cycle during leukemic transformation, progression, and therapeutic resistance of CML. This model can also be used for preliminary drug screenings and investigating the potential for using 
combination therapies targeting cells in specific stages of the cell cycle. Optimization of this protocol establishing the Fucci system in our CML cell line has also advanced work towards using this model in vivo for more physiologically relevant modeling of CML. Work with this system can be used for continued research focusing on overcoming the development of resistance to current CML therapies, which could ultimately lead to identification of a more effective and lasting cure for CML and other CSC-driven malignancies. 
A)

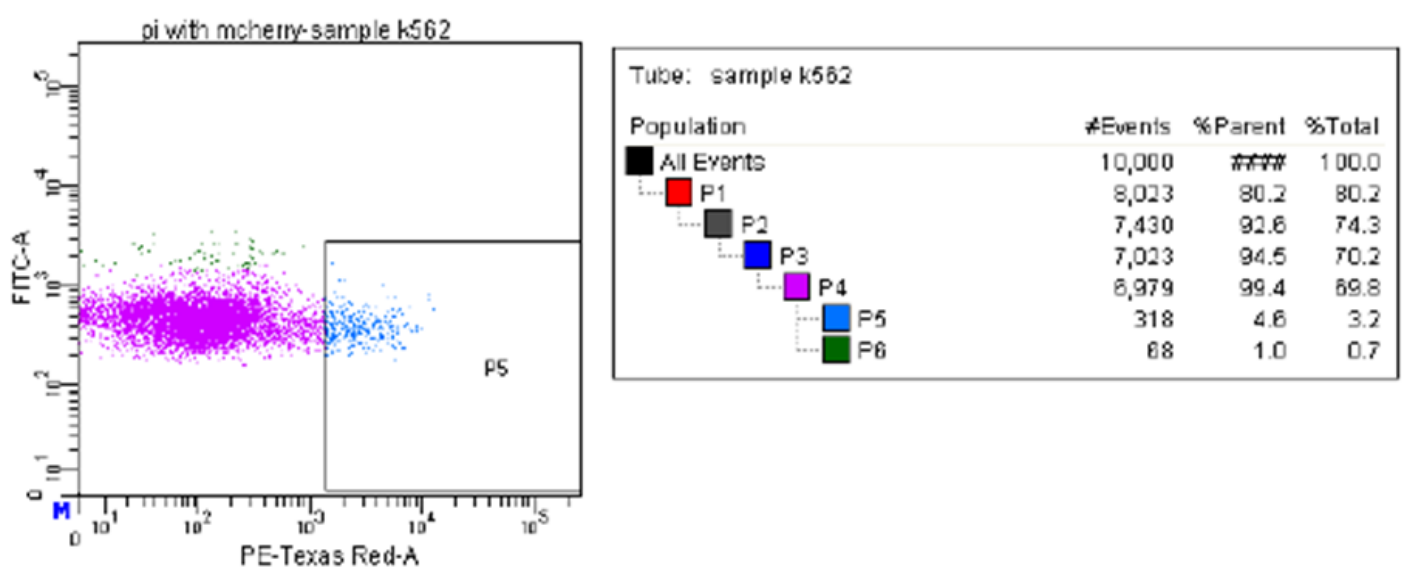

B)
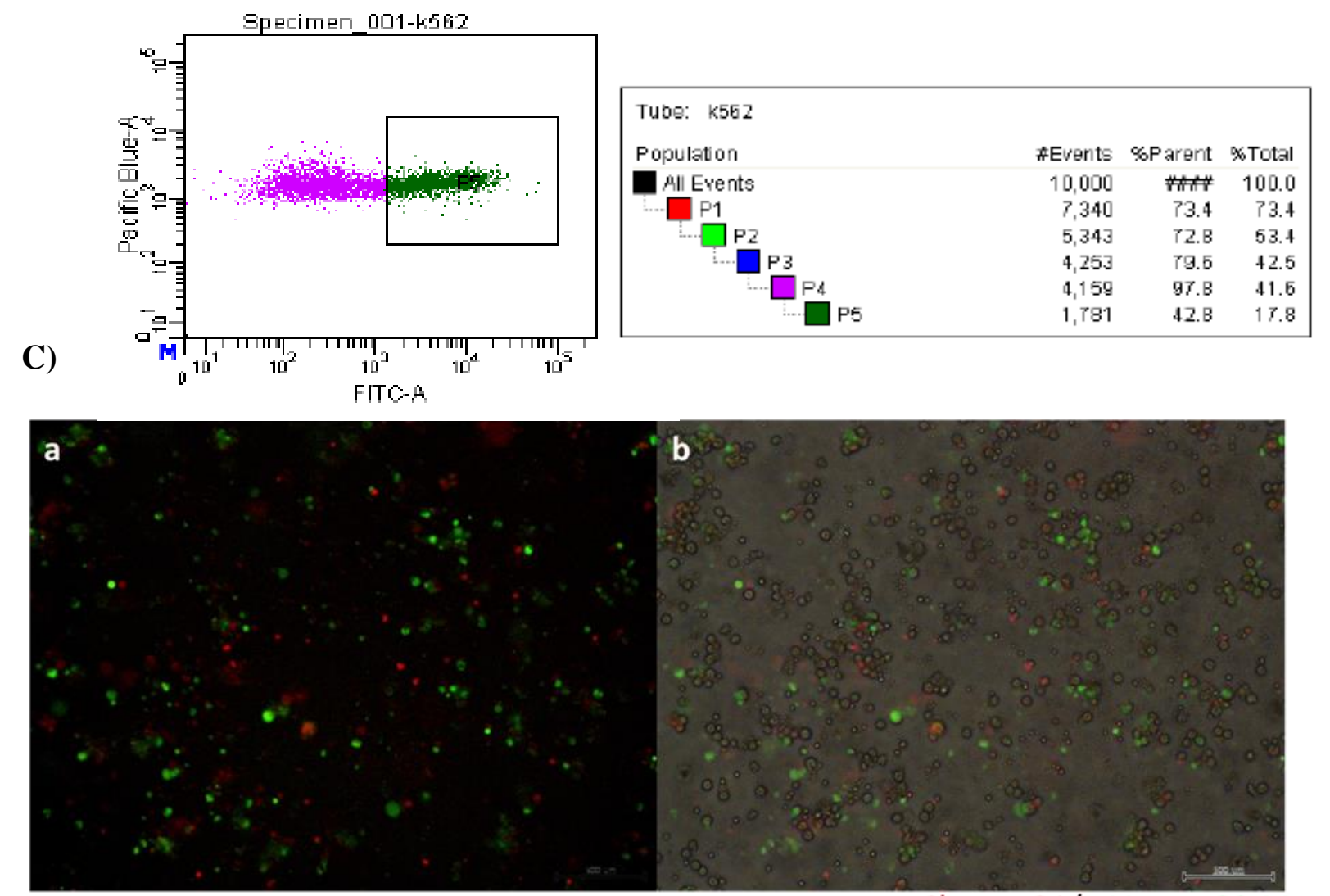

mCherry-G1/mVenus-G2-M

Page 31 
Fig. 1. Establishment of a stably double positive Fucci R-M K562 cell line. K562 cells were expanded in normal culture conditions and transduced with lentiviral Fucci vectors in two series of transductions. A) Fucci-R transduction was performed first, followed by fluorescence sorting and expansion of mCherry positive cells. B) Fucci-R positive cells were then transduced with Fucci-M, and subsequently sorted for mVenus fluorescence expression. C) This population of cells shows retention of fluorescence throughout subculture and expansion as seen by fluorescence microscopy on an Olympus CXK41 with a) showing the overlay of red and green fluorescence and b) the same population with phase contrast. 


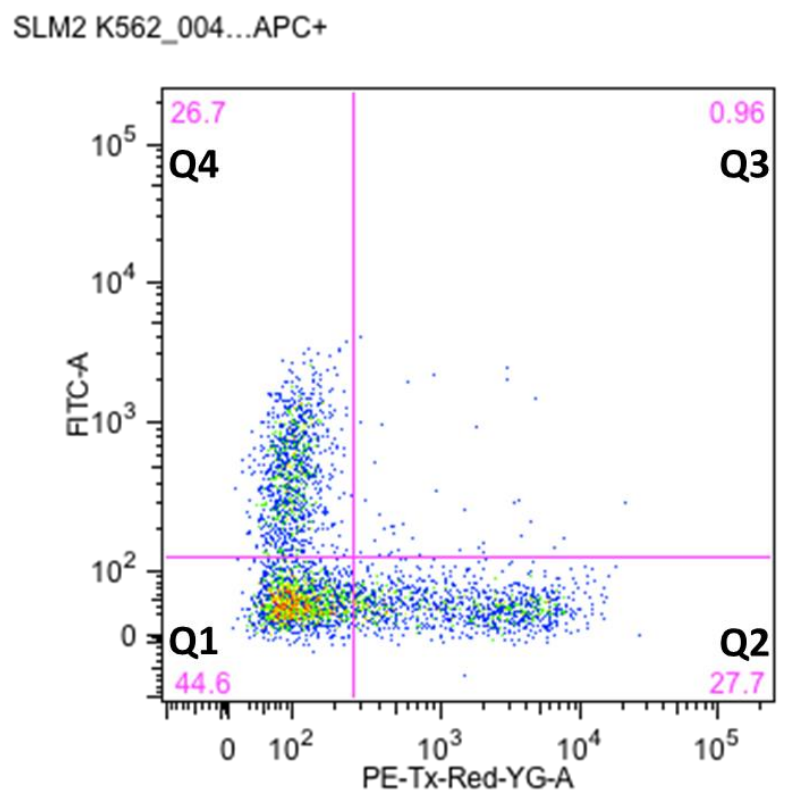

Fig. 2. Analysis of the cell cycle using the Fucci system. This novel Fucci K562 cell line was analyzed based on the fluorescence indicators associated with this system. The $\mathrm{x}$-axis represents the intensity of red fluorescence, whereas the y-axis represents the intensity of green fluorescence. Colorless cells seen in quadrant 1 (Q1) are expressing none of the labeled ubiquitination markers and are therefore in G0 of the cell cycle. Q2 shows cells expressing only mCherry, indicating G1 stage. Double positive cells exhibiting expression of both mCherry and mVenus indicates the overlap of the two fluorophores and the G1/S transition. After the G1/S transition, mCherry expression is lost, and mVenus positive cells indicate G2 as well as M stage (Q4). 


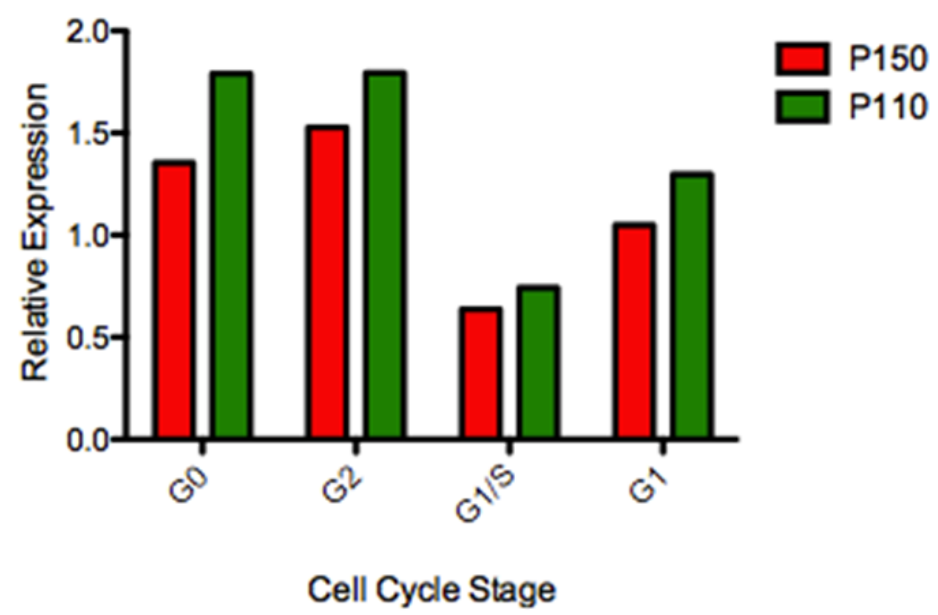

Fig. 3. ADAR1 expression in K562 cells in specific stages of the cell cycle. Double positive K562 Fucci-R/M cells were sorted by FACS for the four stages of the cell cycle based on the properties of the Fucci system. Relative ADAR1 expression was analyzed by RT qPCR using human specific ADAR1 primers for both ADAR1 p150 (P150) and ADAR1 p110 (P110). No qualitative differences in either ADAR1 isoform expression. It appears that there is a decrease in ADAR1 mRNA expression in the G1/S transition, however results are not conclusive. It should be noted that this is just preliminary data validating the Fucci system for use in CML research, and that relative abundance of ADAR1 in different stages of the cell cycle could be different in primary samples as compared to this cell line, due to the fact that $n=1$ for this experiment. The results of this experiment indicate that the Fucci system can be used in conjunction with FACS sorting to generate distinct populations of cells in specific stages of the cell cycle that can be used for subsequent experimentation. 
A)
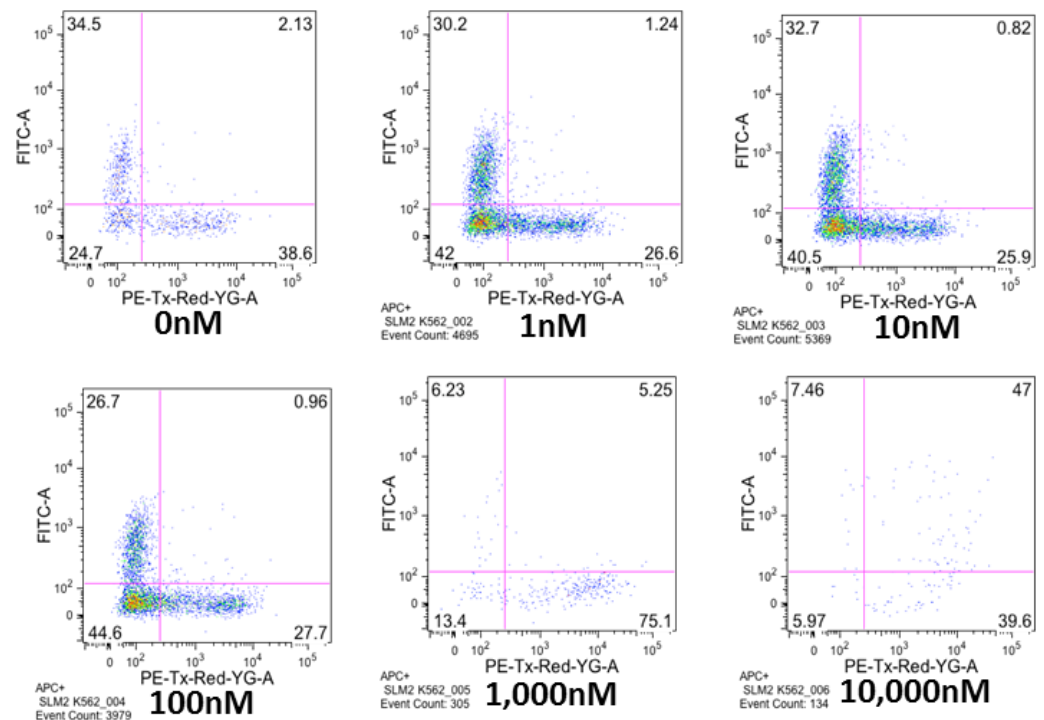

B)

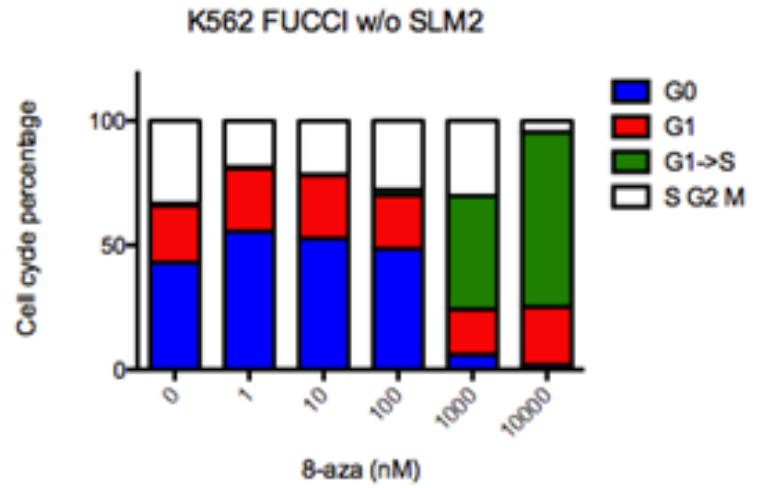

Page 35 


\section{C)}

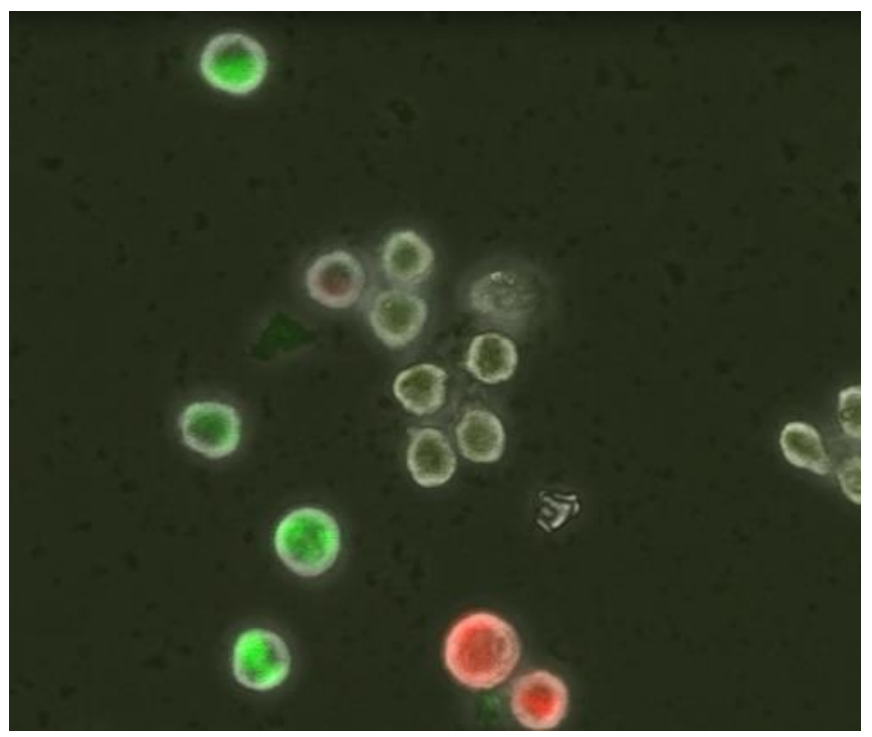

Fig. 4. Effects of 8-azaadenosine titration on cell cycle. K562 Fucci cells were treated with a titration of a novel therapeutic agent, 8-azaadenosine for 5 days before analysis by FACS. Cells were plated in 12-well plates at a density of $5 \times 10^{4}$, treated with each corresponding dose of 8-azaadenosine (or DMSO as a vehicle only treatment). A) FACS plots show the distribution of cells in each stage of the cell cycle. The progression of the cell cycle can be seen in a counter-clockwise progression; quiescent G0 cells show no fluorescence therefore are located in the bottom left quadrant. The reciprocal activity of the two major E3 ubiquitin ligases results in the accumulation of two distinct cell cycle regulatory proteins at different stages of the cell cycle. As cells cycle and express accumulation of the Cdt1 protein in G1 they exhibit red fluorescence. The G1/S transition is marked by the overlap of the two fluorophores. G2 through M phases are marked by the accumulation of the geminin protein and green fluorescence. B) Graph showing the percentages of cells in each stage of the cell cycle. Results seem to indicate 
a shift from quiescence to active cycling. C) Following the titration experiment, cells were subjected to 24 hour treatment with 10,000nM 8-azaadenosine and imaged continuously using a computer assisted Olympus confocal microscope. Images captured at 5 minute intervals allow for visualization of the cells progressing through the cell cycle. Image shown is a still taken from an early time point and shows cells in G1 (red fluorescence) and G2/M (green fluorescence). Dead cells present colorless (as well as displaying not normal morphology), as when the cell membrane becomes permeable upon death, fluorescence is no longer contained in the cell. 


\section{References}

1. Druker, B.J., et al., Efficacy and safety of a specific inhibitor of the BCR-ABL tyrosine kinase in chronic myeloid leukemia. N Engl J Med, 2001. 344(14): p. 1031-7.

2. Salesse, S. and C. Verfaillie, BCR/ABL: from molecular mechanisms of leukemia induction to treatment of chronic myelogenous leukemia. Oncogene, 2002. 21(56): p. 8547-59.

3. Spiers, A.S.D., METAMORPHOSIS OF CHRONIC GRANULOCYTIC LEUKAEMIA: DIAGNOSIS, CLASSIFICATION, AND MANAGEMENT. British Journal of Haematology, 1979. 41(1): p. 1-7.

4. Bonnet, D. and J.E. Dick, Human acute myeloid leukemia is organized as a hierarchy that originates from a primitive hematopoietic cell. Nat Med, 1997. 3(7): p. 730-7.

5. Daley, G., Induction of chronic myelogenous leukemia in mice. Science, 1990. 2406902(824): p. 247.

6. Hochhaus, A., et al., Impact of BCR-ABL mutations on patients with chronic myeloid leukemia. Cell Cycle, 2011. 10(2): p. 250-260.

7. Perrotti, D., et al., Chronic myeloid leukemia: mechanisms of blastic transformation. The Journal of clinical investigation, 2010. 120(7): p. 2254.

8. Nowell, P.C. and D.A. Hungerford, Chromosome Studies on Normal and Leukemic Human Leukocytes. Journal of the National Cancer Institute, 1960. 25(1): p. 85-93, NP1, 94, NP2, 95-109.

9. Rowley, J. Identificaton of a translocation with quinacrine fluorescence in a patient with acute leukemia. in Annales de genetique. 1973.

10. Crews, L.A. and C.H. Jamieson, Chronic myeloid leukemia stem cell biology. Curr Hematol Malig Rep, 2012. 7(2): p. 125-32.

11. Goldman, J.M. and J.V. Melo, Chronic Myeloid Leukemia - Advances in Biology and New Approaches to Treatment. New England Journal of Medicine, 2003. 349(15): p. 1451-1464.

12. Bruns, I., et al., The hematopoietic stem cell in chronic phase CML is characterized by a transcriptional profile resembling normal myeloid progenitor cells and reflecting loss of quiescence. Leukemia, 2009. 23(5): p. 892-9.

13. Nielsen, J.S. and K.M. McNagny, CD34 is a Key Regulator of Hematopoietic Stem Cell Trafficking to Bone Marrow and Mast Cell Progenitor Trafficking in the Periphery. Microcirculation, 2009. 16(6): p. 487-496.

14. Krause, D., et al., CD34: structure, biology, and clinical utility [see comments]. Blood, 1996. 87(1): p. 1-13.

15. Lund, F.E., et al., CD38: a new paradigm in lymphocyte activation and signal transduction. Immunological Reviews, 1998. 161(1): p. 79-93.

16. Funaro, A. and F. Malavasi, Human CD38, a surface receptor, an enzyme, an adhesion molecule and not a simple marker. Journal of biological regulators and homeostatic agents, 1999. 13(1): p. 54. 
17. Partidá-Sánchez, S., et al., CD38: An Ecto-Enzyme at the Crossroads of Innate and Adaptive Immune Responses, in Crossroads between Innate and Adaptive Immunity, P. Katsikis, S. Schoenberger, and B. Pulendran, Editors. 2007, Springer US. p. 171-183.

18. Stuart, S.A., Y. Minami, and J.Y. Wang, The CML stem cell: evolution of the progenitor. Cell Cycle, 2009. 8(9): p. 1338-43.

19. Valent, P., et al., Cancer stem cell definitions and terminology: the devil is in the details. Nature Reviews Cancer, 2012.

20. Park, C., D. Bergsagel, and E. McCulloch, Mouse myeloma tumor stem cells: a primary cell culture assay. Journal of the National Cancer Institute, 1971. 46(2): p. 411-422.

21. Goldman, J.M. and J.V. Melo, BCR-ABL in Chronic Myelogenous LeukemiaHow Does It Work? Acta haematologica, 2008. 119(4): p. 212-217.

22. Lugo, T.G., et al., Tyrosine kinase activity and transformation potency of bcr-abl oncogene products. Science, 1990. 247(4946): p. 1079-1082.

23. Pendergast, A.M., et al., BCR-ABL-induced oncogenesis is mediated by direct interaction with the SH2 domain of the GRB-2 adaptor protein. Cell, 1993. 75(1): p. 175-185.

24. Zou, X. and K. Calame, Signaling Pathways Activated by Oncogenic Forms of Abl Tyrosine Kinase. Journal of Biological Chemistry, 1999. 274(26): p. 1814118144.

25. Fialkow, P.J., S.M. Gartler, and A. Yoshida, Clonal origin of chronic myelocytic leukemia in man. Proceedings of the National Academy of Sciences of the United States of America, 1967. 58(4): p. 1468.

26. Cross, N., et al., BCR-ABL1-positive CML and BCR-ABL1-negative chronic myeloproliferative disorders: some common and contrasting features. Leukemia: official journal of the Leukemia Society of America, Leukemia Research Fund, UK, 2008. 22(11): p. 1975.

27. Bedi, A., et al., Inhibition of apoptosis by BCR-ABL in chronic myeloid leukemia. Blood, 1994. 83(8): p. 2038-2044.

28. Sarry, J.-E., et al., Human acute myelogenous leukemia stem cells are rare and heterogeneous when assayed in NOD/SCID/IL2R $\gamma$ c-deficient mice. The Journal of Clinical Investigation, 2011. 121(1): p. 384-395.

29. Jamieson, C.H.M., et al., Granulocyte-Macrophage Progenitors as Candidate Leukemic Stem Cells in Blast-Crisis CML. New England Journal of Medicine, 2004. 351(7): p. 657-667.

30. Copland, M., et al., Dasatinib (BMS-354825) targets an earlier progenitor population than imatinib in primary CML but does not eliminate the quiescent fraction. Blood, 2006. 107(11): p. 4532-4539.

31. Soverini, S., et al., Philadelphia-positive acute lymphoblastic leukemia patients already harbor BCR-ABL kinase domain mutations at low levels at the time of diagnosis. Haematologica, 2011. 96(4): p. 552-557.

32. Druker, B.J., et al., Effects of a selective inhibitor of the Abl tyrosine kinase on the growth of Bcr-Abl positive cells. Nature medicine, 1996. 2(5): p. 561-566. 
33. Schindler, T., et al., Structural mechanism for STI-571 inhibition of abelson tyrosine kinase. Science Signalling, 2000. 289(5486): p. 1938.

34. Shah, N.P., et al., Overriding imatinib resistance with a novel ABL kinase inhibitor. Science Signalling, 2004. 305(5682): p. 399.

35. Graham, S.M., et al., Primitive, quiescent, Philadelphia-positive stem cells from patients with chronic myeloid leukemia are insensitive to STI571 in vitro. Blood, 2002. 99(1): p. 319-325.

36. Gromicho, M., et al., Development of imatinib and dasatinib resistance: dynamics of expression of drug transporters ABCB1, ABCC1, ABCG2, MVP, and SLC22A1. Leukemia \& Lymphoma, 2011. 52(10): p. 1980-1990.

37. Tokarski, J.S., et al., The structure of dasatinib (BMS-354825) bound to activated ABL kinase domain elucidates its inhibitory activity against imatinib-resistant ABL mutants. Cancer Research, 2006. 66(11): p. 5790-5797.

38. Bhatia, R., et al., Persistence of malignant hematopoietic progenitors in chronic myelogenous leukemia patients in complete cytogenetic remission following imatinib mesylate treatment. Blood, 2003. 101(12): p. 4701-4707.

39. Abrahamsson, A.E., et al., Glycogen synthase kinase $3 \beta$ missplicing contributes to leukemia stem cell generation. Proceedings of the National Academy of Sciences, 2009. 106(10): p. 3925-3929.

40. Pajares, M.J., et al., Alternative splicing: an emerging topic in molecular and clinical oncology. The Lancet Oncology, 2007. 8(4): p. 349-357.

41. Véliz, E.A., L.M. Easterwood, and P.A. Beal, Substrate analogues for an RNAediting adenosine deaminase: Mechanistic investigation and inhibitor design. Journal of the American Chemical Society, 2003. 125(36): p. 10867-10876.

42. Bass, B.L., RNA editing by adenosine deaminases that act on RNA. Annual review of biochemistry, 2002. 71: p. 817.

43. Bass, B.L. and H. Weintraub, A developmentally regulated activity that unwinds RNA duplexes. Cell, 1987. 48(4): p. 607-13.

44. George, C.X. and C.E. Samuel, Human RNA-specific adenosine deaminase ADARI transcripts possess alternative exon 1 structures that initiate from different promoters, one constitutively active and the other interferon inducible. Proceedings of the National Academy of Sciences, 1999. 96(8): p. 4621-4626.

45. Dominissini, D., et al., Adenosine-to-inosine RNA editing meets cancer. Carcinogenesis, 2011.

46. Kim, D.D., et al., Widespread RNA editing of embedded alu elements in the human transcriptome. Genome research, 2004. 14(9): p. 1719-1725.

47. Rueter, S.M., T.R. Dawson, and R.B. Emeson, Regulation of alternative splicing by RNA editing. Nature, 1999. 399(6731): p. 75-80.

48. Lev-Maor, G., et al., Intronic <italic>Alu</italic>s Influence Alternative Splicing. PLoS Genet, 2008. 4(9): p. e1000204.

49. Graveley, B.R., Alternative splicing: increasing diversity in the proteomic world. TRENDS in Genetics, 2001. 17(2): p. 100-107.

50. Osenberg, S., et al., <italic >Alu</italic> Sequences in Undifferentiated Human Embryonic Stem Cells Display High Levels of A-to-I RNA Editing. PLoS ONE, 2010. 5(6): p. e11173. 
51. XuFeng, R., et al., ADARl is required for hematopoietic progenitor cell survival via RNA editing. Proceedings of the National Academy of Sciences, 2009.

106(42): p. 17763-17768.

52. Hartner, J.C., et al., ADAR1 is essential for the maintenance of hematopoiesis and suppression of interferon signaling. Nature immunology, 2008. 10(1): p. 109-115.

53. Cenci, C., et al., Down-regulation of RNA Editing in Pediatric Astrocytomas ADAR2 EDITING ACTIVITY INHIBITS CELL MIGRATION AND PROLIFERATION. Journal of Biological Chemistry, 2008. 283(11): p. 72517260 .

54. Zhang, F. and R. Rabinovici, Adenosine deaminase acting on RNA 1 accelerates cell cycle through increased translation and activity of cyclin-dependent kinase 2. Shock, 2007. 27(2): p. 214-9.

55. Jiang, Q., et al., ADAR1 promotes malignant progenitor reprogramming in chronic myeloid leukemia. Proceedings of the National Academy of Sciences, 2013. 110(3): p. 1041-1046.

56. Phelps, K., A. Morris, and P.A. Beal, Novel modifications in RNA. ACS Chemical Biology, 2011. 7(1): p. 100-109.

57. Wong, S.K., S. Sato, and D.W. Lazinski, Substrate recognition by ADAR1 and ADAR2. RNA, 2001. 7(6): p. 846-858.

58. Lehmann, K.A. and B.L. Bass, Double-Stranded RNA Adenosine Deaminases ADAR1 and ADAR2 Have Overlapping Specificities $†$. Biochemistry, 2000. 39(42): p. 12875-12884.

59. Bennett, L.L., Jr. and P.W. Allan, Metabolism and metabolic effects of 8azainosine and 8-azaadenosine. Cancer Res, 1976. 36(11 Pt 1): p. 3917-23.

60. Glazer, R.I. and L.S. Lloyd, Effects of 8-azaadenosine and formycin on cell lethality and the synthesis and methylation of nucleic acids in human colon carcinoma cells in culture. Biochem Pharmacol, 1982. 31(20): p. 3207-14.

61. Sakaue-Sawano, A., et al., Visualizing Spatiotemporal Dynamics of Multicellular Cell-Cycle Progression. Cell, 2008. 132(3): p. 487-498.

62. Ang, X.L. and J.W. Harper, Interwoven ubiquitination oscillators and control of cell cycle transitions. Science Signaling, 2004. 2004(242): p. pe31.

63. Koepp, D.M., J.W. Harper, and S.J. Elledge, How the Cyclin Became a Minireview Cyclin: Regulated Proteolysis in the Cell Cycle. Cell, 1999. 97: p. 431-434.

64. Nakayama, K.I. and K. Nakayama, Ubiquitin ligases: cell-cycle control and cancer. Nature Reviews Cancer, 2006. 6(5): p. 369-381.

65. Hershko, A. and A. Ciechanover, The ubiquitin system. Annual review of biochemistry, 1998. 67(1): p. 425-479.

66. Sakaue-Sawano, A., et al., Drug-induced cell cycle modulation leading to cellcycle arrest, nuclear mis-segregation, or endoreplication. BMC cell biology, 2011. 12(1): p. 2.

67. Goff, Daniel J., et al., A Pan-BCL2 Inhibitor Renders Bone-Marrow-Resident Human Leukemia Stem Cells Sensitive to Tyrosine Kinase Inhibition. Cell Stem Cell, 2013. 12(3): p. 316-328. 
68. Jelinek, J., et al., JAK2 mutation $1849 G>T$ is rare in acute leukemias but can be found in CMML, Philadelphia chromosome-negative CML, and megakaryocytic leukemia. Blood, 2005. 106(10): p. 3370-3373.

69. Beer, P.A., et al., Two routes to leukemic transformation after a JAK2 mutationpositive myeloproliferative neoplasm. Blood, 2010. 115(14): p. 2891-2900.

70. Li, L. and W.B. Neaves, Normal Stem Cells and Cancer Stem Cells: The Niche Matters. Cancer Research, 2006. 66(9): p. 4553-4557. 